\title{
Fully developed laminar flow of non-Newtonian liquids through annuli: comparison of numerical calculations with experiments
}

\author{
M.P. Escudier, P.J. Oliveira, F.T. Pinho, S. Smith
}

Abstract Experimental data are reported for fully developed laminar flow of a shear-thinning liquid through $K$ both a concentric and an $80 \%$ eccentric annulus with and $n$ without centrebody rotation. The working fluid was an aqueous solution of $0.1 \%$ xanthan gum and $0.1 \%$ carboxymethylcellulose for which the flow curve is well represented by the Cross model. Comparisons are reported between numerical calculations and the flow data, as well as with other laminar annular-flow data for a variety of shear-thinning liquids previously reported in the literature. In general, the calculations are in good quantitative agreement with the experimental data, even in situations where viscoelastic effects, neglected in the calculations, would be expected to play a role.

\section{Abbreviations \\ $D_{\mathrm{H}} \quad$ hydraulic diameter, $2 \delta(\mathrm{m})$ \\ $e$ displacement of inner-cylinder axis from outer-cylinder axis $(\mathrm{m})$ \\ $f \quad$ Fanning friction factor, $-\delta \frac{\partial p}{\partial z} / \rho U^{2}$}

Received: 26 July 2001 / Accepted: 21 February 2002

Published online: 5 June 2002

(C) Springer-Verlag 2002

M.P. Escudier ( $\square)$, S. Smith

Department of Engineering,

Mechanical Engineering,

University of Liverpool, Brownlow Hill,

Liverpool L69 3GH, UK

E-mail: escudier@liv.ac.uk

Tel.: +44-151-7944804

Fax: $+44-151-7944848$

P.J. Oliveira

Departamento de Engenharia Electromecânica,

Universidade da Beira Interior,

Rua Marquês D'Ávila e Boloma,

6200 Covilhã, Portugal

F.T. Pinho

Centro de Estudos de Fenómenos de Transporte,

DEMEGI, Faculdade de Engenharia,

Universidade do Porto, Rua Roberto Frias,

4200-465 Porto, Portugal

The authors gratefully acknowledge the assistance of Drs J.M. Nouri and C. Nouar in providing further details of their published experimental data. The financial support of EPSRC/MTD (research Grant No. GR/F87813), Shell Research BV, and British Gas plc is gratefully acknowledged by M.P. Escudier. P.J. Oliveira and F.T. Pinho acknowledge the receipt of sabbatical leave grants by FCT (Grant No. FMRH/BSAB/68/98 and Grant No. BLS 68/97). The authors' names are listed in alphabetical order. gap width (sector $\mathrm{A}, \mathrm{B}, \mathrm{C}$, or $\mathrm{D})(\mathrm{m})$

consistency index $\left(\mathrm{Pa} \mathrm{s}^{n}\right)$

power-law index

pressure $(\mathrm{Pa})$

radial distance from axis of inner cylinder $(\mathrm{m})$

outer radius of inner cylinder $(\mathrm{m})$

inner radius of outer cylinder $(\mathrm{m})$

bulk axial Reynolds number, $2 \rho U \delta / \mu_{\mathrm{F}}$

Reynolds number for power-law fluid when $\omega=0$

Taylor number, $\left(\rho \omega / \mu_{\mathrm{F}}\right)^{2} R_{\mathrm{I}} \delta^{3}$

$\mathrm{Ta}$

$T a_{0}$

$u$

Taylor number for power-law fluid when $U=0$

axial component of velocity $(\mathrm{m} / \mathrm{s})$

bulk axial velocity $(\mathrm{m} / \mathrm{s})$

tangential component of velocity $(\mathrm{m} / \mathrm{s})$

radial component of velocity $(\mathrm{m} / \mathrm{s})$

radial distance from outer wall of inner cylinder $(\mathrm{m})$

axial distance $(\mathrm{m})$

nondimensional value of $\dot{\gamma}_{\mathrm{F}}, \dot{\gamma}_{\mathrm{F}} \delta / U$

shear rate $\left(\mathrm{s}^{-1}\right)$

characteristic shear rate for flow $\left(\mathrm{s}^{-1}\right)$

mean annular gap width, $R_{\mathrm{O}}-R_{\mathrm{I}}(\mathrm{m})$

eccentricity, $e / \delta$

radius ratio, $R_{\mathrm{I}} / R_{\mathrm{O}}$

dynamic viscosity ( $\mathrm{Pa} \mathrm{s}$ )

characteristic viscosity for flow (Pa s)

viscosity for zero shear rate ( $\mathrm{Pa} \mathrm{s}$ )

viscosity for infinite shear rate ( $\mathrm{Pa} \mathrm{s}$ )

velocity ratio, $\omega R_{\mathrm{I}} / U$

fluid density $\left(\mathrm{kg} / \mathrm{m}^{3}\right)$

nondimensional distance from outer wall

of inner cylinder, $y / g$

shear stress $(\mathrm{Pa})$

yield stress $(\mathrm{Pa})$

angular location with respect to inner cylinder

angular velocity of inner cylinder $(\mathrm{rad} / \mathrm{s})$

\section{Subscripts \\ CR Cross model \\ HB Herschel-Bulkley model \\ PL power-law model}

1

\section{Introduction}

It has been estimated (Pearson 1988) that some $10^{6} \mathrm{~m}^{3}$ of drilling muds are used annually to drill $10^{4}$ or more oil and gas wells. During drilling operations these liquid muds are pumped from a surface mud tank through the drillpipe (several kilometres in length), through nozzles in the rotating drillbit, and back to the mud tank through the

101

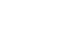


annular space between the wellbore wall and the drillpipe. Drilling muds have several functions: to support the wellbore wall and prevent its collapse, to prevent ingress of formation fluids (gas and liquid) into the wellbore, to transport rock cuttings to the surface, to minimise settling of the cuttings if circulation is interrupted, to clear the workface, to cool the drillbit, and to lubricate the drillstring. These requirements are related directly both to the cost effectiveness of drilling operations and also to their safety, for example, kick detection and control (blowout prevention). The composition of a drilling mud is formulated to meet these requirements, which will differ from well to well. In general, however, drilling muds are colloidal systems that are thixotropic, shear-thinning liquids exhibiting apparent yield stress and some degree of viscoelasticity.

Since the annual cost of the drilling operations outlined above runs into billions of dollars, it comes as no surprise that the oil companies themselves together with the oilfieldservice companies have invested heavily in research into the flow of drilling mud ("mud hydraulics"). The problem that has received most attention from fluid dynamicists has been the calculation of the flowfield within the drillstringwellbore annulus. This situation is usually idealised as that of steady, isothermal, fully developed laminar flow of a generalised Newtonian (shear-thinning) fluid through an annulus consisting of an outer cylindrical cylinder and an inner cylinder, which may be offset (i.e. eccentric) and rotating. In conventional drilling the radius ratio of the annulus geometry is typically about 0.5 , whereas in slimhole drilling and coiled-tube operations it exceeds 0.8 . In reality the problem is complicated by numerous factors: for example, the wellbore wall invariably departs significantly from circularity, the eccentricity varies with depth, the pressure and temperature increase significantly with depth, and drilling muds are to some degree (depending upon the chemical composition) viscoelastic and thixotropic and contaminated with drill cuttings and formation fluid (gas and liquid). However, a complete analysis of the idealised problem is a pre-requisite to a quantitative understanding of these more complex situations.

Although the drilling of oilwells is the most important application, there are numerous other applications that require a detailed understanding of the annular flow of non-Newtonian liquids. Such applications include oilwell-completion operations and are also found in industries dealing with sewage and other industrial waste, with slurries and suspensions such as processed foodstuffs, synthetic fibres and even blood, and with the extrusion of molten plastics and polymer solutions.

Even the idealised problem specified above is far from trivial and it is only very recently that it has been possible to carry out calculations to predict all aspects of the flowfield. The present status has been reached gradually: in a recent paper (Escudier et al. 2002) we list 126 papers published since 1946 dealing with progressively more complex aspects of the problem, initially using analytical techniques (both exact and approximate), followed by numerical solutions to physical approximations, and more recently, purely numerical procedures to solve the complete idealised problem. Escudier et al. 2002 present the results of an extensive series of calculations for powerlaw fluids. They also develop a general methodology by which the power-law results can be applied to flows for fluids obeying other viscosity models (Herschel-Bulkley, Carreau, and Cross) and compare their results for a powerlaw fluid with those of Meuric et al. (1998). The basic computer code implemented by Escudier et al. (2002) is the same as that which they used for the Newtonian case (Escudier et al. 2000), except that the viscosity is replaced by a specified function of the local shear rate.

We are aware of only ten papers that provide experimentally determined velocity distributions for the laminar flow of non-Newtonian liquids through an annulus, and only half of these include the influence of inner cylinder rotation: those of Xisheng and Yinghu (1986), Nouar et al. (1987), Nouri and Whitelaw $(1994,1997)$, and Nouar et al. (1998). This paucity of detailed experimental data may be attributed to the fact that only recently has suitable nonintrusive instrumentation (in the form of laser Doppler anemometry) been commercially available. The limited early measurements of Tiu and Bhattacharyya (1974), for purely axial flow through a concentric annulus, and of Mitsuishi and Aoyagi (1973), for an eccentric annulus, were obtained using particle-tracking techniques. More recent measurements for purely axial flow through a concentric annulus have been reported by Nouar and Lebouché (1996) for Carbopol 940, which is well represented by the Herschel-Bulkley model, and by Nouar et al. (1987) for carboxymethylcellulose (CMC), a near power-law fluid. Xisheng and Yinghu (1986) provided limited data for the helical flow of $0.25 \%$ polyacrylamide through a concentric annulus with the flow modelled as a power-law fluid. Naimi et al. (1990) and Nouar et al. (1998) detail measurements of $0.2 \%$ Carbopol 940 for a concentric annulus with centrebody rotation. Although primarily concerned with turbulent flow, some data for nominally laminar flow of $0.2 \%$ CMC are reported by Nouri et al. (1993) for axial flow in both a concentric and an eccentric annulus, by Nouri and Whitelaw (1994) for a concentric annulus with centrebody rotation, and by Nouri and Whitelaw (1997) for the eccentric case with inner cylinder rotation. Previous experimental results are summarized in Table 1.

The two principal contributions of the present paper (Sect. 4) are to provide extensive new data, which essentially doubles the database, with emphasis on the highly eccentred (80\%) situation and to compare numerical predictions of the flowfield with both the published and the new data. The details of our experimental procedure are given in Sect. 2. The governing equations for the flows under consideration are stated in Sect. 3 and an outline of the numerical procedure is also included, together with remarks on the solution accuracy.

\section{2}

Experimental rig, instrumentation, and test liquid

The flow loop used for our experiments, shown schematically in Fig. 1, is a slightly modified version (to permit eccentring of the inner cylinder) of the annular-flow facility used by Escudier and Gouldson (1995). Flow is 


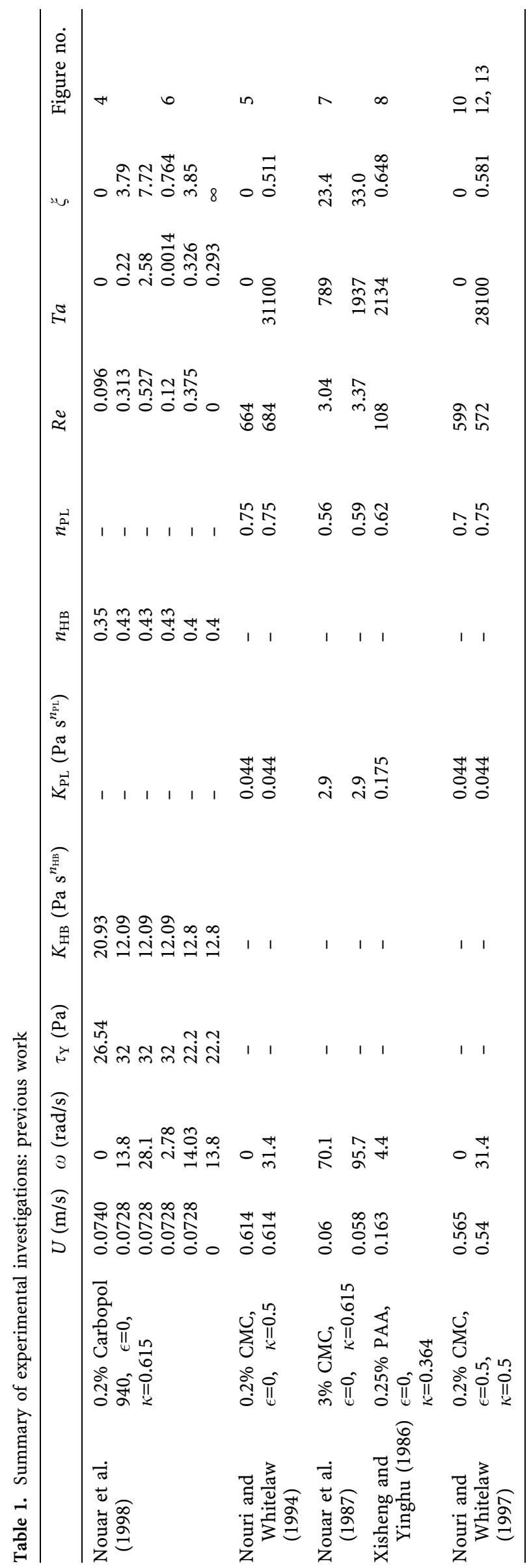

provided by a progressive cavity pump $[1]^{1}$ (Mono-type E101, maximum flowrate $0.025 \mathrm{~m}^{3} / \mathrm{s}$ ) fed directly from a 500-l capacity stainless steel tank of $0.5-\mathrm{m}^{3}$ capacity [2]. Three accumulators [3] located immediately after the pump outlet act to remove pulsations in the flow prior to entry into the test geometry [4]. The annular test section consists of six precision-bore borosilicate glass tubes (ID $100.4 \mathrm{~mm}$ ) with a $50.8-\mathrm{mm}$ diameter stainless steel inner tube [5], giving a radius ratio $\kappa$ of 0.506 . The outer-pipe glass tubes are assembled into modules with male/female stainless steel flanges at alternate ends. The glass of each module is separated from the stainless steel by a PTFE ring, where each end of the module is assembled in a jig and bonded together using Devcon urethane rubber. The complete test section consists of five modules, each of $1.027-\mathrm{m}$ length, and one of $0.64 \mathrm{~m}$, which gives an overall length of $5.775 \mathrm{~m}$ and a length-to-hydraulic diameter ratio of 116. To minimise sagging and vibration of the inner tube, the centrebody wall thickness was selected to provide near neutral buoyancy in the water-based test fluid. In addition, a 3-tonne axial load is applied by means of a hydraulic jack [6]. The inner cylinder can be rotated at any speed up to $126 \mathrm{rpm}$ by means of a DC motor and gearbox. The outer glass tubing is supported on linear bearings and connected to the end housings through sliding plates so that the geometry can be eccentred between $\epsilon=0$ and $\epsilon=1$. For the measurements reported here, $\epsilon$ was either 0 or 0.8 .

Velocities were measured using a laser Doppler anemometer (LDA) system (Dantec Fibreflow) comprising a $60 \times 10$ probe and a $55 \times 12$ beam expander [11] together with a signal processor (Dantec BSA 57N10 Burst Spectrum Analyser). The LDA optical parameters were as follows: beam separation at front lens $51.487 \mathrm{~mm}$, focal length of front lens $160 \mathrm{~mm}$, length of principal axis of measuring volume (in water) $0.180 \mathrm{~mm}$, and length of minor axis of measuring volume (in water) $0.016 \mathrm{~mm}$. All velocity measurements were biased according to residence time. In view of the small size of the measurement volume, it was not felt necessary to make any gradient correction to the measured velocities.

The probe head, housing both the transmitting and receiving optics, was mounted on a three-axis traverse [12]. A flat-faced optical box [13] positioned over the pipe at the measurement location was used to minimise refraction of the beams and hence simplify the refraction correction calculations. Castor oil (refractive index 1.478) was used as the liquid medium between the glass front of the box and the pipe wall. The traversing system, which was controlled by a microcomputer, had a spatial resolution of $15 \mu \mathrm{m}$. Velocity distributions were made at a location 4.5-m (91 hydraulic diameters) downstream of the inlet to the annulus. Measurements for the eccentric geometry were made in two orthogonal planes, along the radii from points $A$ and $C$ (see Fig. 2). For the eccentric arrangement, sector A of the annulus is referred to as the widest gap, $\mathrm{C}$ as the narrowest gap, and $\mathrm{D}$ as the widening gap. In practice, measurements in the narrowest and widening gaps were made with the centre of the inner

${ }^{1}$ The numbers in square brackets refer to the components shown in Fig. 1. 


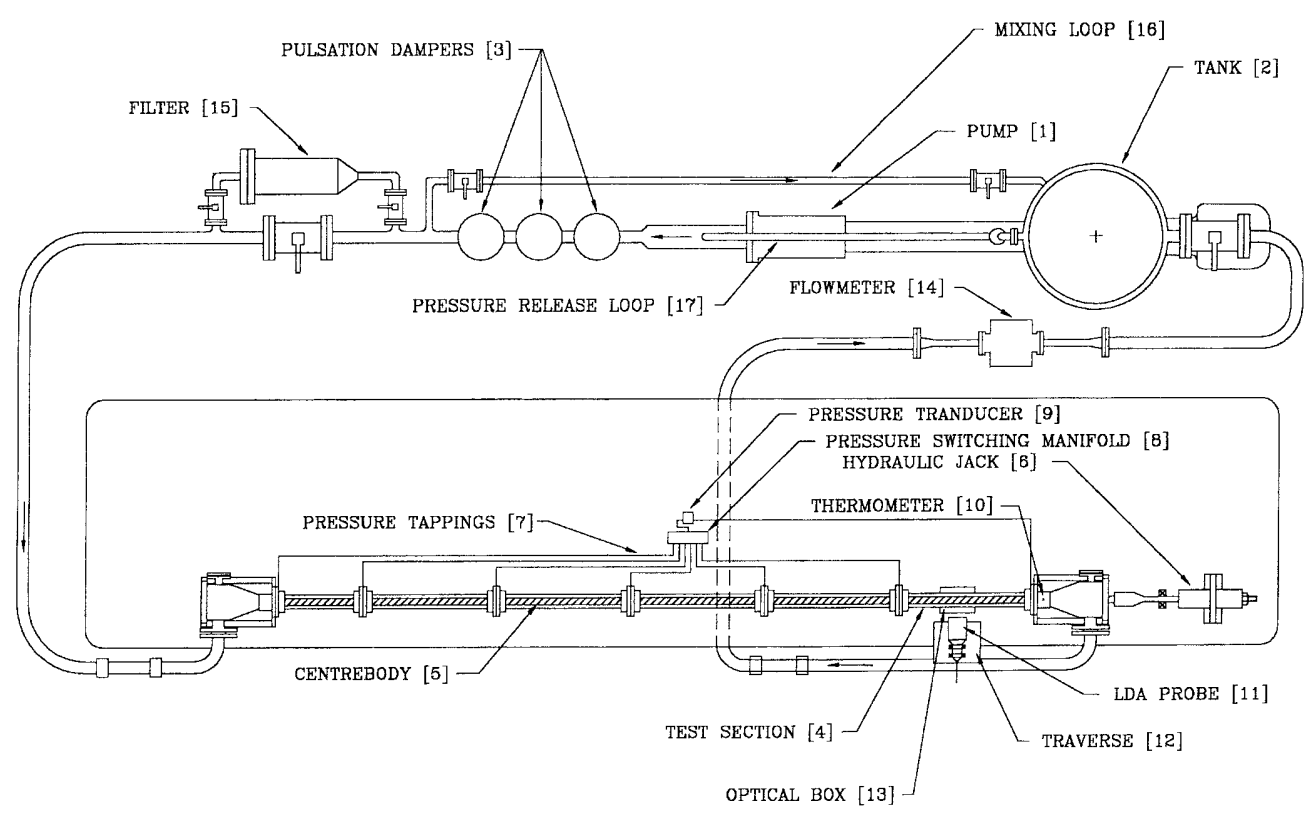

Fig. 1. Schematic diagram of flow loop (plan view)

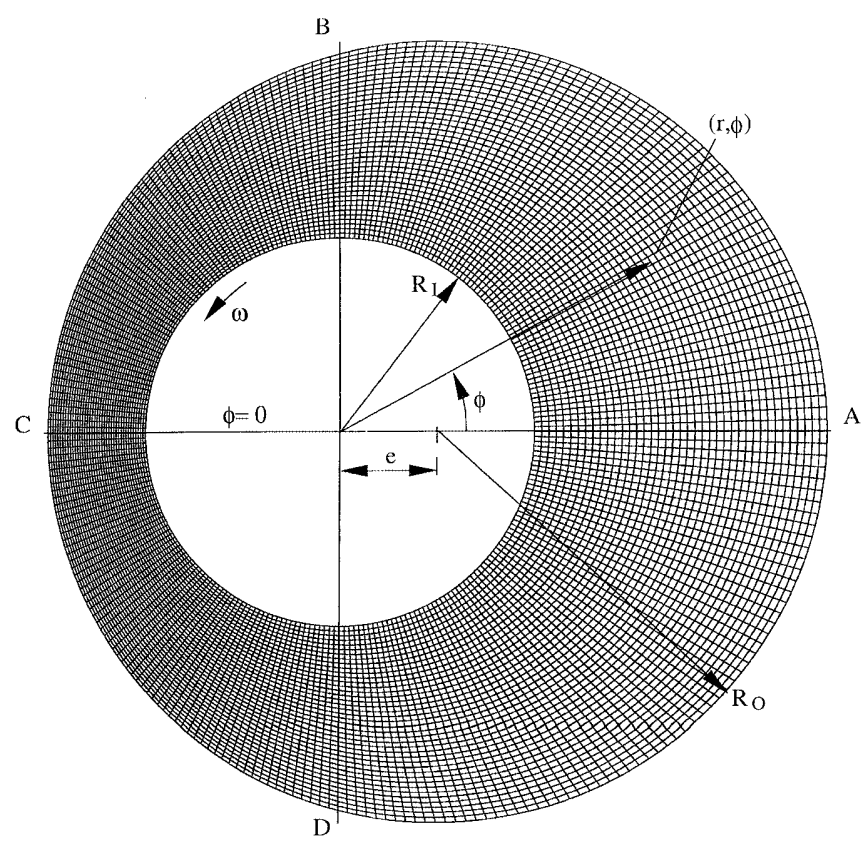

Fig. 2. Annulus geometry and computational grid $(40 \times 256$ cells)

cylinder located to the right of the centre of the outer cylinder rather than to its left as shown in Fig. 2. For the axial-flow measurements, positional corrections were required to account for refraction at the air-glass-liquid boundaries, whilst for the tangential component corrections to both position and velocity were necessary. The detailed flow measurements are discussed in Sect. 4.

An electromagnetic flowmeter [14] (Fischer and Porter model $10 \mathrm{D} 1)$ is incorporated in the return arm of the flow loop, with the output signal recorded via an analog-todigital converter (Amplicon PS $30 \mathrm{AT}$ ) on a PC (IBM AT286). Flow rates indicated by the flowmeter were within $1 \%$ of values computed from velocity profiles measured in pipe flow (Escudier and Presti 1996) using the LDA system, which is a measure of the overall accuracy of the measurements. In-house software was written to record flowrate, pressure drop, and fluid temperature and to control and record traverse location.

To permit filtering of the base solvent (tap water) prior to the addition of the polymers, a $125-\mu \mathrm{m}$ filter [15] is incorporated into the bypass loop through which the flow can be diverted. Mixing of the polymers is accomplished by circulating the fluid through a return loop [16] to the tank incorporated just after the pulsation dampers. A pressure-relief (safety) valve and return loop [17] are located immediately after the pump outlet.

The test liquid was a blend of $0.1 \%$ xanthan gum (XG) and $0.1 \%$ carboxymethylcellulose (CMC) for which a typical flow curve is shown in Fig. 3. The CMC was a high-viscosity grade supplied by the Aldrich Chemical Company and the XG a food-grade polymer supplied by the Kelco Division of Merck. The viscometric characteristics of this fluid were determined using a controlledstrain rheometer (Bohlin VOR) with cone-and-plate and

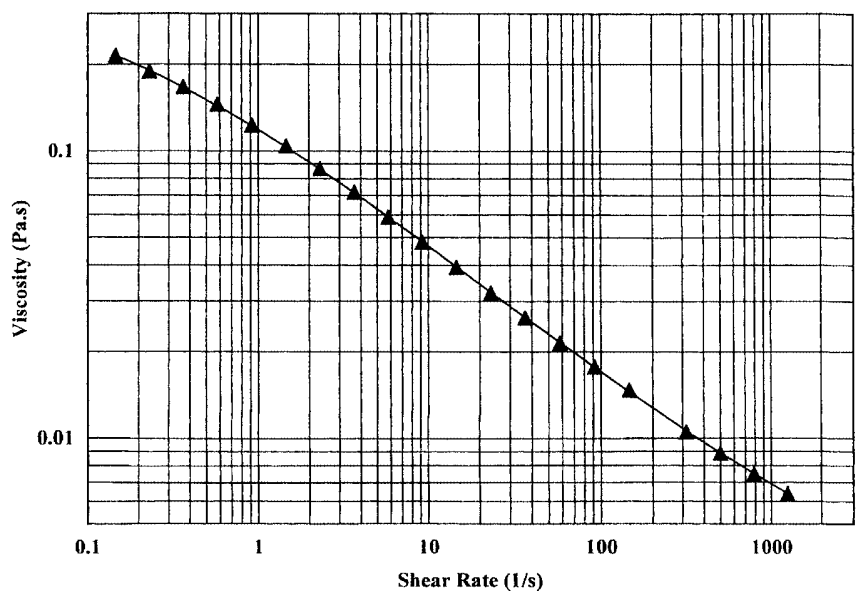

Fig. 3. Flow curve for $0.1 \%$ xanthan gum $/ 0.1 \%$ carboxmethylcellulose (CMC) blend 
parallel-plate geometries. As can be seen, this $\mathrm{CMC} / \mathrm{XG}$ blend is close to being a power-law fluid, but the viscometric data are better fitted by the Cross model (Cross 1965), i.e.

$\frac{\mu_{\mathrm{o}}-\mu_{\infty}}{\mu-\mu_{\infty}}=1+K_{\mathrm{CR}} \dot{\gamma}^{n_{\mathrm{CR}}}$

Values for the consistency index $K_{\mathrm{CR}}$ and the exponent $n_{\mathrm{CR}}$ are listed in Table 2 together with the global parameters (see Sect. 3.2) for each experiment.

At the concentration used for the flow experiments, the first normal-stress differences for $\mathrm{CMC} / \mathrm{XG}$ are below the sensitivity of standard laboratory rheometers even at the highest shear rates. However, as suggested by Barnes et al. (1989), at higher concentrations it was found that $N_{1}(\tau)$ data followed a power-law master curve for each fluid, practically independently of concentration, from which it was possible to extrapolate to lower concentrations. This procedure is not ideal but has to suffice in the absence of either a direct measurement or a more sophisticated extrapolation algorithm. The data obtained using a controlled-stress rheometer (TA Instruments Rheolyst AR $1000 \mathrm{~N}$, Escudier et al. 1999) result in the empirical expression

$N_{1}=1.35 \tau^{1.18}, \quad \mathrm{CMC} / \mathrm{XG} \quad 0.4 / 0.4 \%-1.5 / 1.5 \%$.

According to Barnes et al., a recoverable shear (i.e. $N_{1} / 2 \tau$ ) greater than 0.5 indicates a highly elastic state, a condition for CMC/XG that corresponds to shear stresses in excess of $0.2 \mathrm{~Pa}$.

The refractive index of the test liquid was measured using an high-accuracy refractometer (ABBE 60/ED).

\section{3}

Governing equations, nondimensional parameters, viscosity models, and numerical procedure

\section{1}

\section{Governing equations}

We consider isothermal, laminar, fully developed flows of fluids for which the density is constant and the viscosity dependent only on the second invariant of the strain-rate tensor (i.e. generalised Newtonian fluids). The governing equations for such flows can be written as follows:

continuity

$\frac{\partial}{\partial r}(w r)+\frac{\partial v}{\partial \phi}=0$

axial momentum

$\rho\left(w \frac{\partial u}{\partial r}+\frac{v}{r} \frac{\partial u}{\partial \phi}\right)=-\frac{\partial p}{\partial z}+\frac{1}{r} \frac{\partial}{\partial r}\left(\mu r \frac{\partial u}{\partial r}\right)+\frac{1}{r^{2}} \frac{\partial}{\partial \phi}\left(\mu \frac{\partial u}{\partial \phi}\right)$

tangential momentum

$$
\begin{aligned}
\rho\left(w \frac{\partial v}{\partial r}+\frac{v}{r} \frac{\partial v}{\partial \phi}+\frac{w v}{r}\right)= & -\frac{1}{r} \frac{\partial p}{\partial \phi}+\frac{1}{r^{2}} \frac{\partial}{\partial r}\left[\mu r^{3} \frac{\partial}{\partial r}\left(\frac{v}{r}\right)+\mu r \frac{\partial w}{\partial \phi}\right] \\
& +\frac{2}{r} \frac{\partial}{\partial \phi}\left(\frac{\mu}{r} \frac{\partial v}{\partial \phi}+\frac{\mu w}{r}\right)
\end{aligned}
$$

radial momentum

$$
\begin{aligned}
\rho\left(w \frac{\partial w}{\partial r}+\frac{v}{r} \frac{\partial w}{\partial \phi}-\frac{v^{2}}{r}\right)= & -\frac{\partial p}{\partial r}+\frac{2}{r} \frac{\partial}{\partial r}\left(\mu r \frac{\partial w}{\partial r}\right)+\frac{\partial}{\partial \phi}\left[\mu \frac{\partial}{\partial r}\left(\frac{v}{r}\right)\right] \\
& +\frac{1}{r^{2}} \frac{\partial}{\partial \phi}\left(\mu \frac{\partial w}{\partial \phi}\right),
\end{aligned}
$$

with boundary conditions $u=v=w=0$ on the outer cylinder, and $u=w=0$ and $v=\omega R_{\mathrm{I}}$ on the inner cylinder.

The magnitude of the rate-of-strain tensor (or shear rate) $\dot{\gamma}$ for fully developed flow is given by

$\dot{\gamma}^{2}=\left[r \frac{\partial}{\partial r}\left(\frac{v}{r}\right)+\frac{1}{r} \frac{\partial w}{\partial \phi}\right]^{2}+\frac{1}{r^{2}}\left(\frac{\partial u}{\partial \phi}\right)^{2}+\left(\frac{\partial u}{\partial r}\right)^{2}+4\left(\frac{\partial w}{\partial r}\right)^{2}$.

It is clear from (4), (5), and (6) that spatial derivatives of the viscosity $\mu$ must be accounted for and that variations with the angular location $\phi$ arise as a consequence of

\begin{tabular}{|c|c|c|c|c|c|c|c|c|c|c|}
\hline $\begin{array}{l}0.1 \% \text { XG/0.1\% } \\
\mathrm{CMC}, \kappa=0.506\end{array}$ & $U(\mathrm{~m} / \mathrm{s})$ & $\omega(\mathrm{rad} / \mathrm{s})$ & $\mu_{0}$ (Pa s) & $\mu_{\infty} \quad(\mathrm{Pa} s)$ & $K_{\mathrm{CR}}\left(\mathrm{s}^{n_{\mathrm{CR}}}\right)$ & $n_{\mathrm{CR}}$ & $R e$ & $\mathrm{Ta}$ & $\xi$ & Figure no. \\
\hline$\epsilon=0$ & 0.203 & 5.24 & 0.159 & 0.00273 & 1.305 & 0.509 & 236 & 6020 & 0.656 & 9 \\
\hline \multirow[t]{9}{*}{$\epsilon=0.8$} & 0.267 & 0 & 0.134 & 0.00290 & 0.540 & 0.530 & 263 & 0 & 0 & \multirow[t]{3}{*}{11} \\
\hline & 0.288 & 0 & 0.116 & 0.00349 & 0.502 & 0.553 & 332 & 0 & 0 & \\
\hline & 0 & 5.31 & 0.134 & 0.00290 & 0.540 & 0.530 & 0 & 2754 & $\infty$ & \\
\hline & 0.268 & 5.17 & 0.174 & 0.00242 & 0.672 & 0.539 & - & - & 0.490 & \multirow[t]{6}{*}{14,15} \\
\hline & 0.268 & 5.33 & - & - & - & - & - & - & 0.505 & \\
\hline & 0.268 & 5.35 & 0.177 & 0.00255 & 0.630 & 0.551 & 225 & 3172 & 0.507 & \\
\hline & 0.268 & 5.20 & 0.174 & 0.00242 & 0.672 & 0.539 & - & - & 0.493 & \\
\hline & 0.268 & 5.20 & - & - & - & - & - & - & 0.493 & \\
\hline & 0.268 & 5.24 & 0.262 & 0.00144 & 2.414 & 0.504 & 241 & 3500 & 0.497 & \\
\hline
\end{tabular}
the eccentricity of the annulus $\epsilon$ (i.e. although the flow is two-dimensional, all three velocity components are

Table 2. Summary of experimental investigation: present work 
nonzero). In Sect. 3.3 we give details of the three viscosity models used in the comparisons with experimental data.

\section{2}

\section{Nondimensional parameters}

For consistency with the Newtonian situation, we define the nondimensional flow parameters:

axial Reynolds number

$R e \equiv \frac{2 \rho U \delta}{\mu_{\mathrm{F}}}$,

and Taylor number

$$
T a \equiv\left(\frac{\rho \omega}{\mu_{\mathrm{F}}}\right)^{2} R_{\mathrm{I}} \delta^{3},
$$

where $\mu_{\mathrm{F}}$ is the viscosity evaluated at a characteristic shear rate for the flow $\dot{\gamma}_{\mathrm{F}}$, defined by

$$
\begin{aligned}
\dot{\gamma}_{\mathrm{F}}^{2} & =\left(\frac{U}{D_{\mathrm{H}}}\right)^{2}+\left(\frac{\omega R_{\mathrm{I}}}{D_{\mathrm{H}}}\right)^{2}=\frac{1}{4}\left[\left(\frac{U}{\delta}\right)^{2}+\left(\frac{\omega R_{\mathrm{I}}}{\delta}\right)^{2}\right] \\
& =\left(\frac{U}{D_{\mathrm{H}}}\right)^{2}\left(1+\xi^{2}\right),
\end{aligned}
$$

and the velocity ratio

$\xi \equiv \frac{\omega R_{\mathrm{I}}}{U}$.

It can be seen that $\xi$ is a direct measure of the nondimensional characteristic shear rate for the flow $\gamma_{\mathrm{F}}$, defined by

$\gamma_{\mathrm{F}}=\frac{\dot{\gamma}_{\mathrm{F}} \delta}{U}=\frac{1}{2} \sqrt{\left(1+\xi^{2}\right)}$.

Our choice for $\dot{\gamma}_{\mathrm{F}}$ is consistent with the work of Lockett (1992), whereas Meuric et al. (1998) adopted different scaling for flows with and without centrebody rotation. This definition has the advantage that the corresponding values of $R e$ and $T a$ reflect to some extent the non-Newtonian coupling between the axial and tangential velocity distributions.

\section{3}

\section{Viscosity models}

The published experimental data with which we compare the results of our numerical calculations were obtained using a range of liquids (all aqueous polymer solutions) with substantially different viscometric and other rheological characteristics. Xisheng and Yinghu (1986) used $0.25 \%$ polyacrylamide (PAA), a viscoelastic liquid, which they represented with a power-law model

$\mu=K_{\mathrm{PL}} \dot{\gamma}^{n_{\mathrm{PL}}-1}$.

Nouri and Whitelaw $(1994,1997)$ also adopted the power law for their experiments with $0.2 \%$ CMC, which is both less elastic than PAA and also less shear-thinning (i.e. higher $n_{\mathrm{PL}}$ ). Nouar et al. (1987) used CMC at a much higher concentration (3\%), which they also modelled as a power-law fluid.
Nouar et al. (1998), on the other hand, adopted the Herschel-Bulkley model for their experiments with $0.2 \%$ Carbopol

$\tau=\tau_{\mathrm{Y}}+K_{\mathrm{HB}} \dot{\gamma}^{n_{\mathrm{HB}}}$.

As stated in Sect. 2, the working fluid for the new experimental data that we present here (a blend of $0.1 \%$ CMC and $0.1 \% \mathrm{XG}$ ) is well represented by the Cross model, see (1).

For the three viscometric models adopted here, values for the two flow parameters $R e$ and $T a$ can be calculated as illustrated by the power-law model for which we have: for $\omega=0$

$R e_{0}=\frac{\rho U^{2-n_{\mathrm{PL}}} D_{\mathrm{H}}^{n_{\mathrm{PL}}}}{K_{\mathrm{PL}}}$,

for $U=0$

$T a_{0}=\frac{1}{8}\left(\rho \frac{\omega^{2-n_{\mathrm{PL}}}}{K_{\mathrm{PL}}}\right)^{2} D_{\mathrm{H}}^{2 n_{\mathrm{PL}}+1} R_{\mathrm{I}}^{3-2 n_{\mathrm{PL}}}$,

and in the general case of combined rotation and throughflow we have:

$R e=\left(1+\xi^{2}\right)^{\frac{\left(1-n_{\mathrm{PL}}\right)}{2}} R e_{0}$,

$T a=\left(\frac{1}{\xi^{2}}+1\right)^{1-n_{\mathrm{PL}}} T a_{0}$.

\section{4}

\section{Numerical procedure}

To calculate the velocity components, the governing equations were transformed into a general nonorthogonal coordinate system. These transformed equations were then discretised following the finite-volume approach of Patankar (1980), but adapted for collocated nonorthogonal grids, as described in Oliveira (1992). The calculations were carried out using a second-order central differencing scheme, and the deferred correction approach was used in order to ensure numerical stability for the convective terms. The solution algorithm was a modified version of the SIMPLEC algorithm of van Doormal and Raithby (1984) adapted for time marching as explained in Issa and Oliveira (1994), where details can be found of the particular procedure used to evaluate mass fluxes at cell faces. When performing calculations with the Herschel-Bulkley model (i.e. for a fluid with a yield stress), the biviscosity model was used to avoid stiffness in the matrices at low shear rates and numerical singularities in nonyielded regions following the criteria suggested by O'Donovan and Tanner (1984).

The coordinate system was centred at the inner cylinder axis and the three-dimensional annular geometry represented by 16 structured blocks around the annulus. A cross section of the annular geometry and the grid arrangement for a typical numerical calculation are shown in Fig. 2. Since the fully developed flow condition was of concern here, only one row of cells $\left(\approx D_{\mathrm{H}}\right.$ in length) was 
needed in the axial direction, and the procedure of Patankar and Spalding (1972) was adopted to correct the axial pressure gradient $\partial p / \partial z$. In the other two directions the grid used in the calculations had $40 \times 256$ cells uniformly distributed in the radial and tangential directions. This grid was selected after a systematic grid-refinement study was performed to assess the accuracy of the calculation using four progressively finer grids: $10 \times 64,20 \times 128$, $40 \times 256$ (as shown in Fig. 2), and $80 \times 512$ cells with Newtonian fluids (Escudier et al. 2000) and extended by Escudier et al. (2002) to power-law fluids.

Numerical accuracy was estimated on the basis of Newtonian and non-Newtonian calculations for concentric and eccentric flow cases and by comparison with the available analytical solutions and, in their absence, with values determined from using Richardson's extrapolationto-the-limit technique applied to the results of simulations with the above-mentioned three finer meshes. For more details the reader is referred to Escudier et al. $(2000,2002)$. For the Newtonian concentric flow, the simulations with grid 3 ( $40 \times 256$ cells) predicted $f \cdot R e$ within $0.1 \%$ of the analytical theory. For power-law fluids having a power-law index $n_{\mathrm{PL}}$ of 0.5 , calculations were performed for a concentric and a $95 \%$ eccentric annulus at $R e=100$ and $T a=10,000$. In this case, the assessment of the accuracy based on the extrapolation technique was found to remain fairly constant and also at about $0.1 \%$.

\section{4}

\section{Comparisons with experiment}

Tables 1 and 2 list all the data with which we compare the results of our calculations: previously published data in Table 1 and our own data in Table 2. Included in the tables are values of the parameters for the appropriate viscosity models together with values of the bulk mean velocity $U$, the rotational speed $\omega$, and the nondimensional parameters $\epsilon, \kappa, R e, T a$, and $\xi$. Perhaps the most striking feature of Tables 1 and 2 is the very wide range of values for both $R e$ and $T a$, given that all are associated nominally with fully developed laminar flow. Since there are so few data sets and there are uncertainties associated both with the velocity measurements and the fluid rheology, we have made comparisons with all the data we are aware of, including the case of the concentric annulus, which can be regarded as a control. In the remaining figures, the solid lines represent the maths of the numerical calculations.

\section{1}

\section{Concentric annulus, no rotation}

The obvious qualitative differences between the two sets of comparisons shown for the simplest situation under consideration, that of flow through a concentric annulus with no rotation of the centrebody (i.e. $T a=0$ ), are a consequence of differences in the fluid rheology and in the Reynolds numbers. The data of Nouar et al. (1998) (Fig. 4) are for $0.2 \%$ Carbopol, a Herschel-Bulkley (i.e. yieldstress) fluid, at very low Reynolds number $(\mathrm{Re}=0.096)$, which results in a flat velocity distribution with a "plastic" central plug. The experiment of Nouri and Whitelaw (1994) (Fig. 5), on the other hand, is for $0.2 \%$ CMC, a power-law fluid with only a modest shear-thinning index

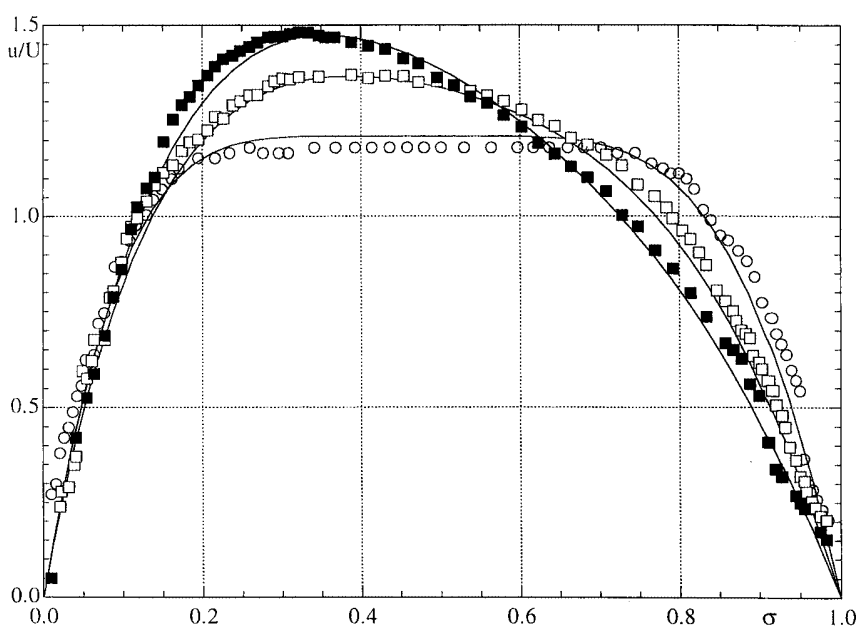

Fig. 4. Axial velocity data of Nouar et al. (1998) for $0.2 \%$ Carbopol 940, $\kappa=0.615, \epsilon=0: R e=0.096, T a=0(\bigcirc) ; R e=0.313, T a=0.22(\square)$;

$R e=0.527, T a=2.58(\mathbf{\square})$

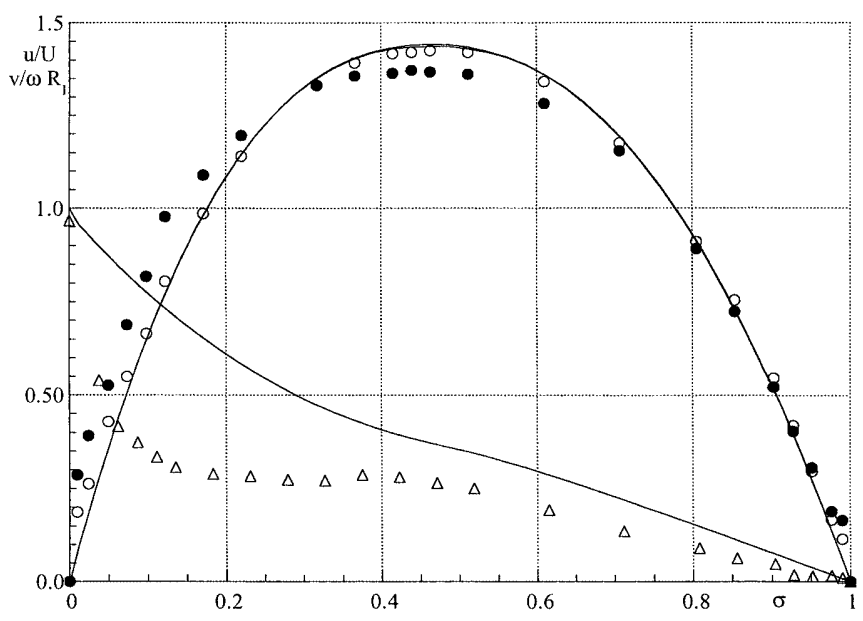

Fig. 5. Data of Nouri and Whitelaw (1994) for $0.2 \%$ CMC, $\kappa=0.5, \epsilon=0$ : $R e=664, T a=0(\bigcirc) ; R e=684, T a=31,100(\bigcirc$, axial $),(\Delta$, tangential)

$(n=0.75)$ and a much higher Reynolds number $(R e=664)$ so that the velocity profile is only slightly flattened compared with that for a Newtonian fluid (i.e. peak velocity $1.44 U$ compared with $1.51 U$ ). The agreement between the experiments and the calculations in these cases is clearly satisfactory.

\section{2}

\section{Concentric annulus with centrebody rotation} and bulk axial flow

Four published sets of data for $\epsilon=0$ are available for comparison purposes. The data of Nouar et al. (1998) for helical flow (Figs. 4, 6) again correspond to very low Re and $T a$ values. The calculations capture all the essential features shown by the experiments, including the radial shift of the peak axial velocity towards the centrebody, the sigmoidal form of the tangential velocity at the lowest Taylor number, and the monotonic distributions at the higher Taylor numbers. Although the qualitative behaviour of the data of Nouar et al. (1987) (Fig. 7) is well 


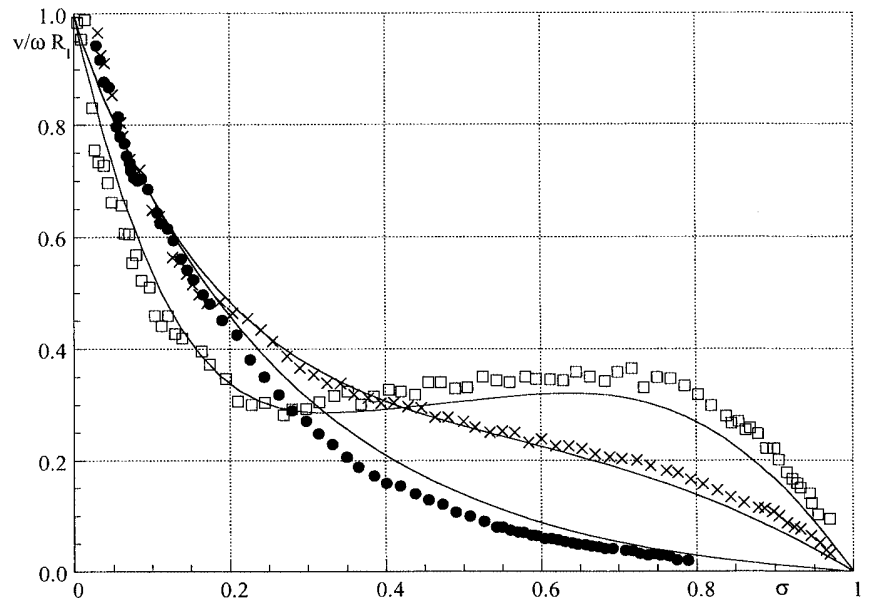

Fig. 6. Tangential velocity data of Nouar et al. (1998) for $0.2 \%$ Carbopol 940, $\kappa=0.615, \epsilon=0: R e=0, T a=0.293(\bullet) ; R e=0.12, T a=0.0014$ ( $\square) ; R e=0.375, T a=0.326(\mathrm{X})$

represented by the calculations, there are minor quantitative differences such as in the location of the peak axial velocity. These data, for a relatively high $(3 \%)$ concentration of CMC, which is both more shear-thinning and more elastic than the Carbopol used by Nouar et al. (1998), correspond to slightly higher $R e$ values and to much higher Taylor numbers.

The data of Nouri and Whitelaw (1994) (Fig. 5) are also for CMC, but at a much lower concentration (0.2\%), and so for a fluid that is less viscous, less shear-thinning and also less elastic. The Reynolds and Taylor numbers for this set of data are the highest of any we consider. According to the calculations, the effect of rotation on the axial velocity distribution is negligible: the two curves are almost indistinguishable and show practically Newtonian behaviour, whereas the experiment shows a significantly flatter axial velocity profile for the situation with rotation. The agreement here is quite poor, particularly for the tangential velocity, and it may well be that the data are affected by Taylor instability or are transitional rather than laminar. Certainly the latter proved to be the case for the data of

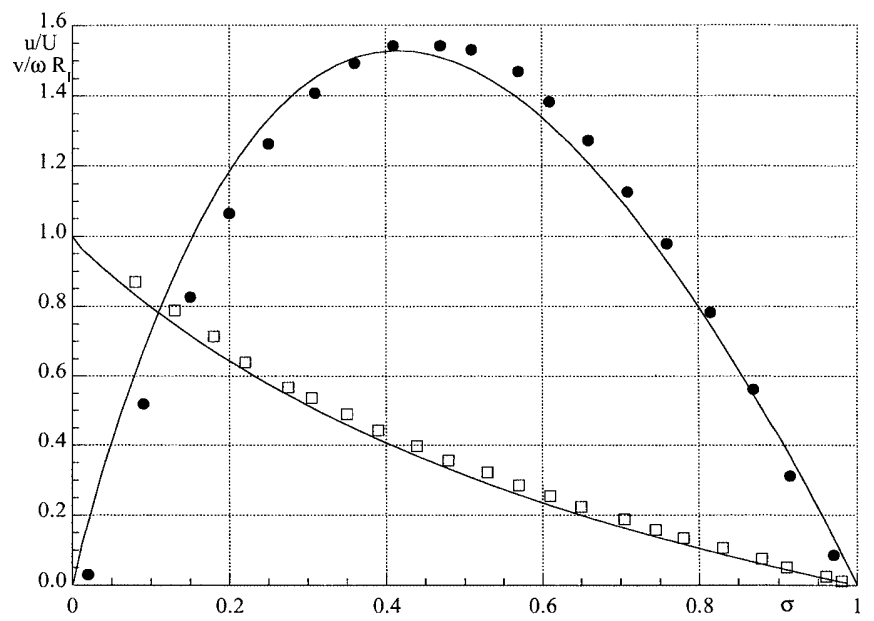

Fig. 7. Data of Nouar et al. (1987) for $3 \%$ CMC, $\kappa=0.615, \epsilon=0$ : $R e=3.04, T a=789(\bigcirc)$; $R e=3.37, T a=1937(\square)$
Escudier and Gouldson (1998), for which $R e=655$ and $T a=12580$, values comparable with those of Nouri and Whitelaw. Our calculations are in very good agreement with the single axial velocity distribution of Xisheng and Yinghu (1986), Fig. 8, which corresponds to Re and Ta values much higher than those of Nouar et al. $(1987,1998)$, but lower than those of Nouri and Whitelaw (1994).

The final set of comparisons (Fig. 9) for flow through a concentric annulus are for our own experiments carried out with $0.1 \%$ XG/0.1\% CMC. Previous work (Escudier et al. 1999) showed this fluid to be slightly less shearthinning but considerably more elastic than either CMC or $\mathrm{XG}$ alone, although less elastic than PAA. There is extremely good agreement with the calculations, which are for Reynolds and Taylor numbers considerably higher than those of Nouar et al. (1987, 1998), but somewhat lower than those of Nouri and Whitelaw (1994), for both the axial and the tangential velocity distributions, except for the behaviour of the tangential velocity in the near vicinity of the centrebody $(\sigma=0)$. Reflections of the laser beams of the LDA system are the probable cause of uncertainty in this region. Both the calculations and the experimental data show practically no differences between the two sets of data, even though the values of $T a$ differ by a factor of almost three.

\section{3}

\section{Eccentric annulus, no rotation}

For the 0.2\% CMC data of Nouri and Whitelaw (1997) (Fig. 10), for which $\epsilon=0.5$, the calculations show a somewhat different distribution of the flow around the annulus (sector A in Fig. 2) than is indicated by the experimental data. In the central region of the widest part of the annulus the calculated velocity exceeds the measured values by about $13 \%$. This is compensated for by a progressive azimuthal decrease into the narrowest part of the annulus (sector C), where the calculated values are only $26 \%$ of those measured. The calculations here lead to results only slightly different from those of Escudier et al. (2000) for a Newtonian fluid. For the shear-thinning fluid, the calculated velocity distributions are all slightly flatter with

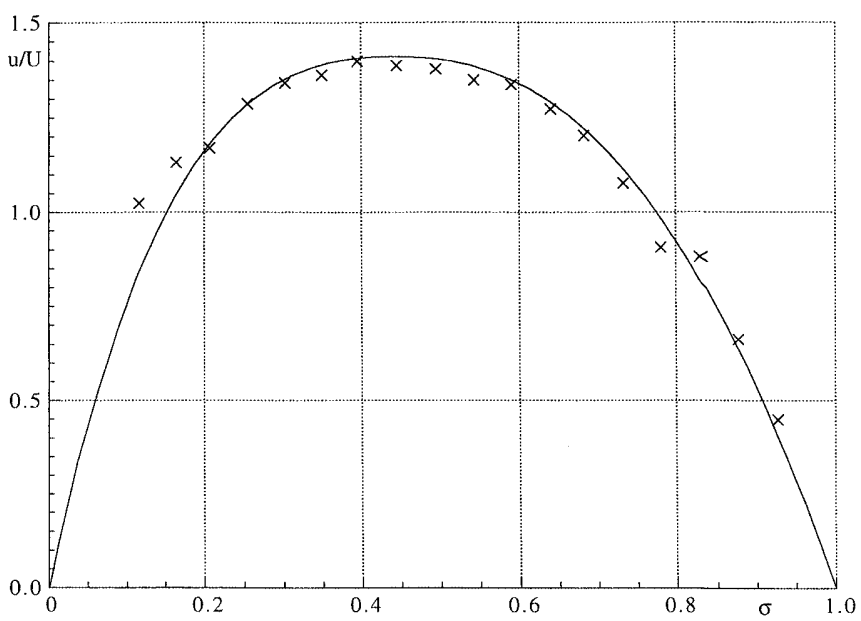

Fig. 8. Axial velocity data of Xisheng and Yinghu (1986) for $0.25 \%$ PAA, $\kappa=0.364, \epsilon=0: R e=108, T a=2,134$ 


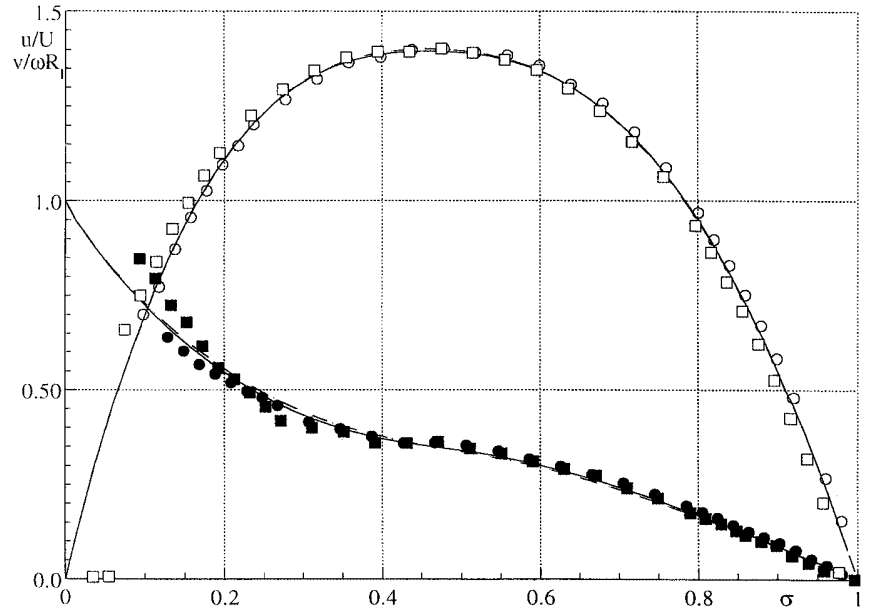

Fig. 9. Data of present authors for $0.1 \% \mathrm{XG} / 0.1 \% \mathrm{CMC}, \kappa=0.506, \epsilon=0$ : $R e=228, T a=2,026$, solid line ( $\square$, axial), ( $\square$, tangential); $R e=236$, $T a=6,020$, dashed line $(\bigcirc$, axial), $(\bullet$, tangential)

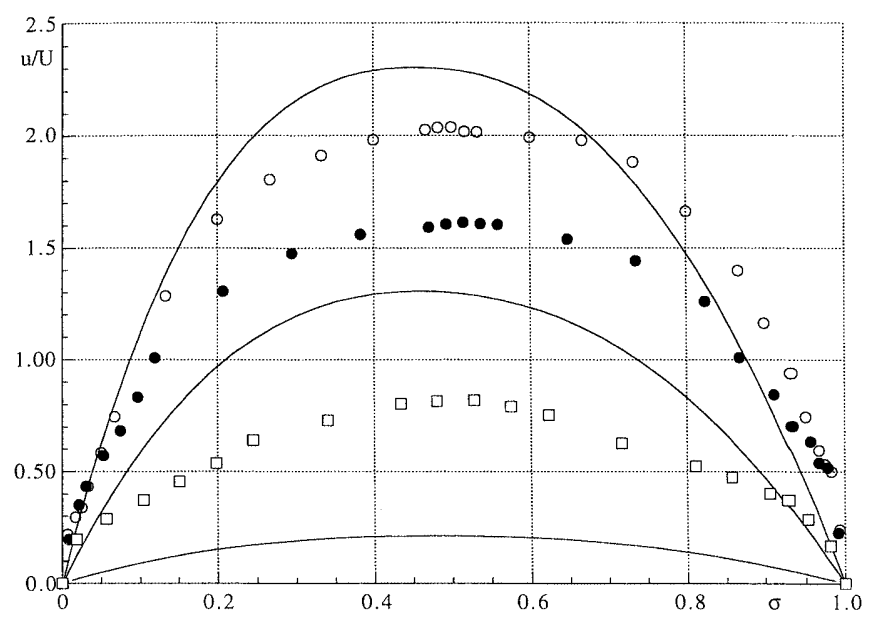

Fig. 10. Axial velocity data of Nouri and Whitelaw (1997) for $0.2 \%$ CMC, $\kappa=0.5, \epsilon=0.5, R e=599, T a=0$ : $(\bigcirc)$ sector A; $(\bullet)$ sector B; $(\square)$ sector $\mathrm{C}$

reduced peak velocities. The experiments of Nouri and Whitelaw show this trend only in the widest part of the annulus, whereas in the narrowest part the velocities are far in excess of the Newtonian values, a characteristic unlikely to be correct for a shear-thinning fluid. It may be that once again these data are not in the vortex-free laminar regime.

The comparisons with our own data $(0.1 \% \mathrm{CMC} / 0.1 \%$ XG) for $\epsilon=0.8$ (Fig. 11) are more satisfactory than those of Fig. 10. In the wide gap (A) there is almost perfect agreement in the region between the outer wall of the annulus $(\sigma=1)$ and $\sigma=0.3$. As for the concentric geometry, there is an increasing discrepancy with approach to the inner cylinder $(\sigma=0)$. For the intermediate gap (B) the profile shape is well predicted, but the measured velocities are about $9 \%$ lower than those calculated. Since there is an appreciable azimuthal gradient of the axial velocity, a slight misalignment of the LDA probe could have led to a velocity distribution that is either slightly too high or too low, which is the probable explanation for the discrepancies in the intermediate gap.

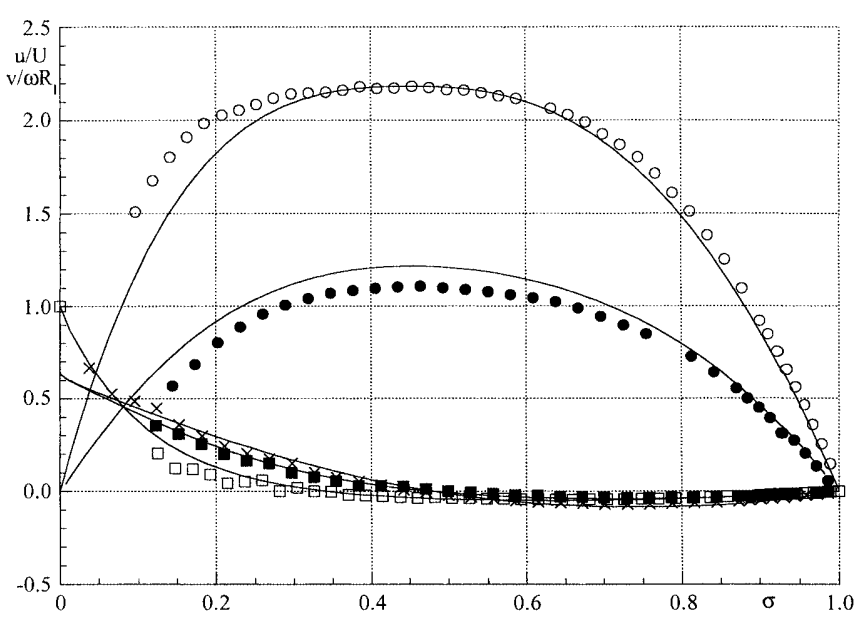

Fig. 11. Data of present authors, $\kappa=0.506, \epsilon=0.8: R e=332, T a=0(\bigcirc)$ axial, sector A; $R e=263, T a=0(\bullet)$ axial, sector B; $R e=0, T a=3,315$ (ם) tangential, sector $\mathrm{A} ; R e=0, T a=2,754(\Phi)$ tangential, sector $\mathrm{B} ; R e=0$, $T a=2,954$ (X) tangential, sector C

\section{4}

\section{Eccentric annulus with rotation, no throughflow}

None of the investigators listed in Table 1 report data for the situation in which flow is induced around an eccentric annulus by inner cylinder rotation in the absence of bulk axial flow. The comparisons with numerical computations therefore are limited to our own data (Fig. 11) for $\epsilon=0.8, T a \approx 3000$, again for $0.1 \% \mathrm{CMC} / 0.1 \%$ XG. The calculations are generally in good agreement with the data, particularly with regard to the magnitude and extent of recirculation $(v<0)$, which is most pronounced in sector B.

\section{5}

\section{Eccentric annulus, with rotation and throughflow}

The only published data of which we are aware for the most complex situation of an eccentric annulus with inner cylinder rotation and bulk throughflow are those of Nouri and Whitelaw (1997) for 0.2\% CMC, $\epsilon=0.5, R e=572$, and $T a=28100$ (Figs. 12, 13). Although there are discrepancies between the calculations and the experimental data, the general level of agreement is better than for the same geometry in the absence of centrebody rotation and not significantly worse than for Nouri and Whitelaw's (1994) data for flow through a concentric annulus. The largest discrepancy is for the axial velocity in the widening gap (D) and could be a consequence of the alignment difficulty mentioned in Sect. 4.3. An interesting feature of the calculations, albeit inconsistent with the experimental data, is the tendency for the axial velocity in the narrow gap (C) to exceed that in sector $\mathrm{D}$. This behaviour is attributable to shear thinning.

For our own experiments, again for $0.1 \% \mathrm{XG} / 0.1 \% \mathrm{CMC}$ and $\epsilon=0.8$ (Figs. 14, 15), the calculations capture all the qualitative features revealed by the data. The greatest discrepancies in comparison with the tangential velocities are in the near vicinity of the inner cylinder for the wide gap (A) and the narrow gap (C), and for the axial velocity in the widening gap (D), again possibly due to the difficulty of alignment. For example, in the narrow gap the tangential velocity must approach $\omega R_{\mathrm{I}}$ as $\sigma$ tends to zero. 


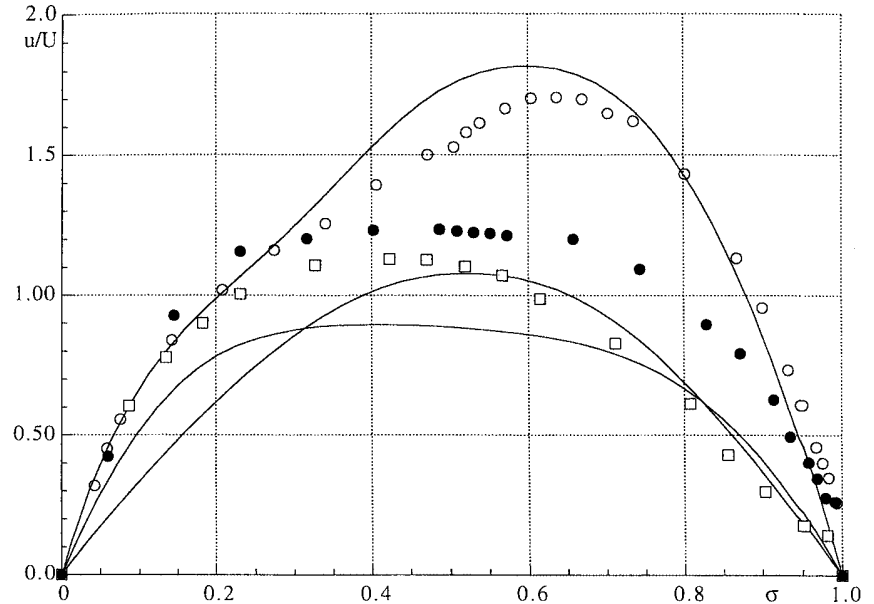

Fig. 12. Axial velocity data of Nouri and Whitelaw (1997) for $032 \%$ CMC, $\kappa=0.5, \epsilon=0.5, R e=572, T a=28,100$ : (O) sector A; $\square$ ) sector C; (O) sector $\mathrm{D}$

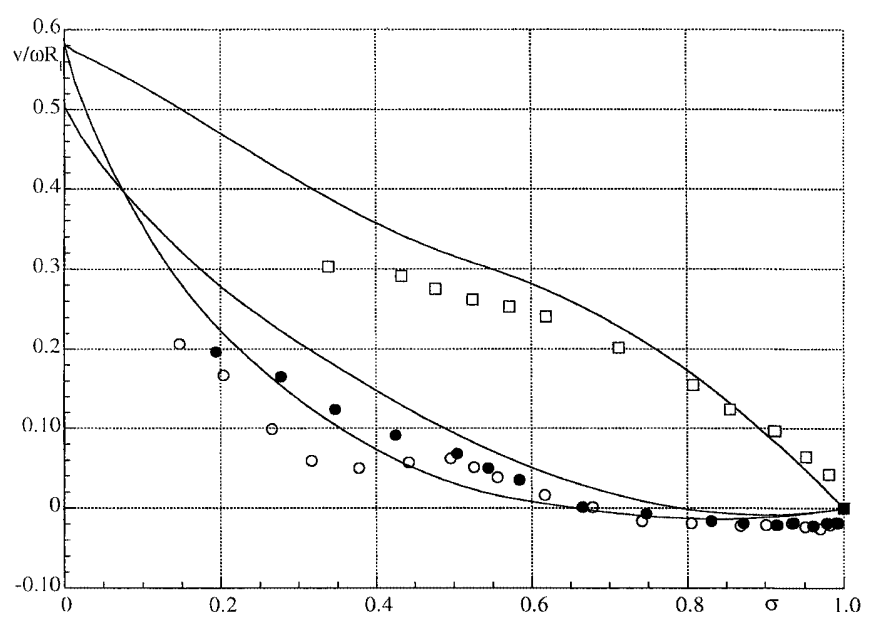

Fig. 13. Tangential velocity data of Nouri and Whitelaw (1997) $\kappa=0.5, \epsilon=0.5, R e=572, T a=28,100$ : $(\bigcirc)$ Sector A; $(\square)$ Sector C; ( Sector D

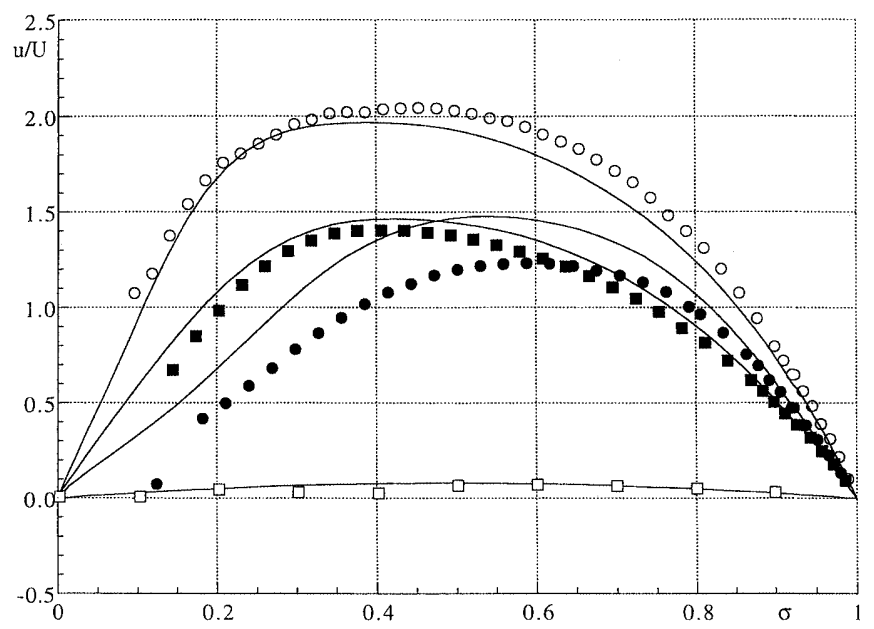

Fig. 14. Axial velocity data of present authors $\kappa=0.506, \epsilon=0.8$ : $R e=241, T a=3,500(\bigcirc)$ sector A; $R e=225, T a=3,172(\square)$ sector B; $(\square)$ sector C; $(\mathbf{O})$ Sector D

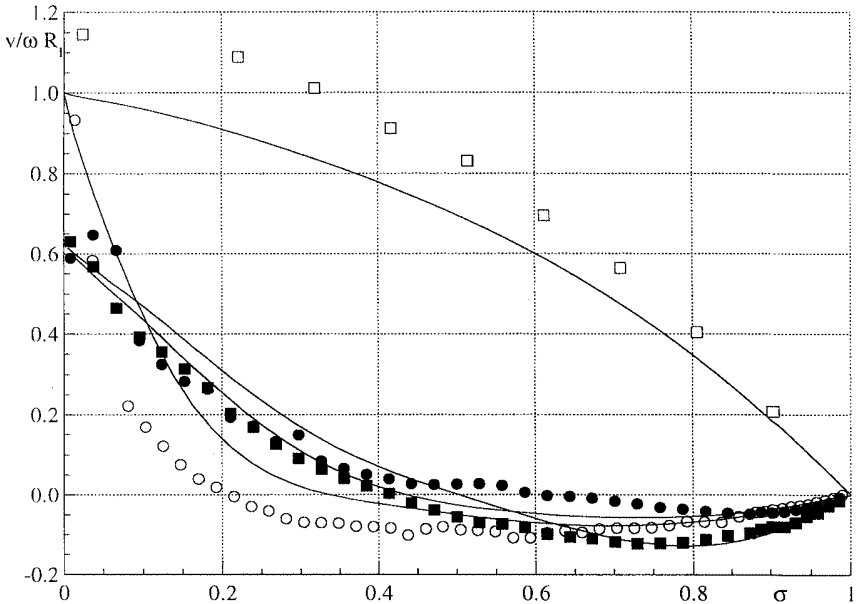

Fig. 15. Tangential velocity data of present authors $\kappa=0.506, \epsilon=0.8$ : $R e=241, T a=3,500(\bigcirc)$ sector A; $R e=225, T a=3,172$ (ם) sector B, ( $\square$ ) sector $\mathrm{C},(\mathbf{)})$ sector $\mathrm{D}$

\section{5}

\section{Concluding remarks}

For all published data, the calculations have been carried out using the inelastic viscosity model proposed by the original investigators. As demonstrated by Escudier et al. (2002) the calculations are relatively insensitive to minor changes to these models. Nevertheless, some of the discrepancies noted in Sect. 4 are undoubtedly associated with the oversimplification represented by the power-law model adopted by Nouar et al. (1987), Nouri and Whitelaw $(1994,1997)$ and by Xisheng and Yinghu (1986). However, in general, this is not expected since, even for steady fully developed flow, the shear-viscosity variation for a viscoelastic fluid will depend on the distribution of the axial elastic normal stress for a concentric annular geometry, and on the two normal stress differences for the eccentric case. Also, it should not be forgotten that the calculations did not take into account viscoelastic effects from either the normal stress or the elongational viscosity. It cannot be taken for granted that an inelastic viscosity model will be adequate in all circumstances even for the calculation of axisymmetric flows, which are regarded as what Astarita and Marucci (1974) term viscometric. As Alves et al. (2001) have shown in a recent paper, the crucial issue is whether the viscosity function adopted for the inelastic model captures the exact shear viscosity behaviour of a fluid that is influenced by elastic normal stresses. It can be concluded that this is the case for the polyacrylamide data of Xisheng and Yinghu (1986). In the case of Nouar et al, for example, the power-law representation is only valid for shear rates in the range 100 to $1200 \mathrm{~s}^{-1}$. Viscoelasticity would be expected to affect any flow in an eccentric annulus (see e.g. Davies and Li 1994), such as those considered in Sect. 4.4, whereas the elongational viscosity only plays a role for the eccentric annulus with inner cylinder rotation, such as those considered in Sect. 4.5. The comparisons of Sect. 4 suggest that for the laminar flows under consideration here these elastic effects can be regarded as negligible. Although some non-Newtonian fluids, such as clays and muds, exhibit thixotropy, there is no evidence that this is the case for any of the fluids considered here, 
and so this influence cannot be seen as a contributory factor for any of the discrepancies identified.

\section{References}

Alves MA, Pinho FT, Oliveira PJ (2001) Theoretical study of steady duct flows of the full Phan-Thien-Tanner fluid. J Non-Newt Fluid Mech 101:55-76

Astarita G, Marucci G (1974) Principles of non-Newtonian fluid mechanics. McGraw-Hill, London

Barnes HA, Hutton JF, Walters K (1989) An introduction to Rheology. Elsevier, Amsterdam

Cross MM (1965) Rheology of non-Newtonian fluids: a new flow equation for pseudoplastic systems. J Colloid Sci 20:417-437

Davies AR, Li XK (1994) Numerical modelling of pressure and temperature effects in viscoelastic flow between eccentrically rotating cylinders. J Non-Newt Fluid Mech 54:331-350

van Doormal JP, Raithby GD (1984) Enhancements of the SIMPLE method for predicting incompressible fluid flows. Num Heat Transfer 7:147-163

Escudier MP, Gouldson IW (1995) Concentric annular flow with centrebody rotation of a Newtonian and a shear-thinning liquid. Int J Heat Fluid Flow 16:156-162

Escudier MP, Gouldson IW (1998) Effects of centrebody rotation on the laminar flow of a shear-thinning liquid through an eccentric annulus. In: Proc. 9th International Symposium on Applications of Laser Techniques to Fluid Mechanics, Lisbon, Portugal, 13-16 July, pp 8.3.1-8.3.7

Escudier MP, Presti F (1996) Pipe flow of a thixotropic liquid. J NonNewt Fluid Mech 62:291-306

Escudier MP, Presti F, Smith S (1999) Drag reduction in the turbulent pipe flow of polymers. J Non-Newt Fluid Mech 81:197-213

Escudier MP, Gouldson IW, Oliveira PJ, Pinho FT (2000) Effects of inner cylinder rotation on laminar flow of a Newtonian fluid through an eccentric annulus. Int J Heat Fluid Flow 21:92-103

Escudier MP, Oliveira PJ, Pinho FT (2002) Fully developed laminar flow of purely viscous non-Newtonian liquids through annuli, including the effects of eccentricity and inner-cylinder rotation. Int J Heat Fluid Flow 23:52-73

Issa RI, Oliveira PJ (1994) Numerical predictions of phase separation in two-phase flow through T-junctions. Comp Fluids 23:347-372

Locket TJ (1992) Numerical simulation of inelastic non-Newtonian fluid flows in annuli. PhD thesis, University of London

Meuric OFJ, Wakeman RJ, Chiu TW, Fisher KA (1998) Numerical flow simulation of viscoplastic fluids in annuli. Can J Chem Eng 76: $27-40$
Mitsuishi N, Aoyagi Y (1973) Non-Newtonian fluid flow in an eccentric annulus. J Chem Eng Japan 6:402-408

Naimi M, Devienne R, Lebouché M (1990) Etude dynamique et thermique de l'écoulement de Couette-Taylor-Poiseuille; cas d'un fluide présentant un seuil d'écoulement. Int J Heat Mass Trans 33:381-391

Nouar C, Desaubry C, Zenaidi H (1998) Numerical and experimental investigation of thermal convection for a thermodependent Herschel-Bulkley fluid in an annular duct with rotating inner cylinder. Eur J Mech B 17:875-900

Nouar C, Devienne R, Lebouché M (1987) Convection thermique pour l'écoulement de Couette avec debit axial: cas d'un fluide pseudoplastique. Int J Heat Mass Trans 30:639-647

Nouar C, Lebouché M (1996) Thermal convection for a thermodependent Herschel-Bulkley fluid in an annular duct. Heat Mass Trans 31:257-267

Nouri JM, Umur H, Whitelaw JH (1993) Flow of Newtonian and nonNewtonian fluids in concentric and eccentric annuli. J Fluid Mech 253:617-641

Nouri JM, Whitelaw JH (1994) Flow of Newtonian and non-Newtonian fluids in a concentric annulus with rotation of the inner cylinder. J Fluids Eng 116:821-827

Nouri JM, Whitelaw JH (1997) Flow of Newtonian and non-Newtonian fluids in an eccentric annulus with rotation of the inner cylinder. Int J Heat Fluid Flow 18:236-246

O’Donovan EJ, Tanner RI (1984) Numerical study of the Bingham squeeze-film problem. J Non-Newt Fluid Mech 15:75-83

Oliveira PJ (1992) Computer modelling of multidimensional multiphase flow and application to T-junctions. $\mathrm{PhD}$ thesis, Imperial College of Science, Technology and Medicine, London

Patankar SV (1980) Numerical heat transfer and fluid flow. Hemisphere, New York

Patankar SV, Spalding DB (1972) A calculation procedure for heat, mass and momentum transfer in three-dimensional parabolic flows. Int J Heat Mass Trans 15:1787-1806

Pearson JRA (1988) Rheological principles and measurements applied to the problems of drilling and completing oil wells. In: Proc. Xth Int Cong Rheology, vol 1, Sydney, 14-19 August, pp 73-78

Tiu C, Bhattacharyya S (1974) Developing and fully developed velocity profiles for inelastic power-law fluids in an annulus. AIChE J 20:1140-1144

Xisheng L, Yinghu Z (1986) An analysis of properties of laminar flow field of power-law fluid in annular space. In: Proc Int Meeting on Petroleum Engineering, Beijing, China, paper SPE 14870 